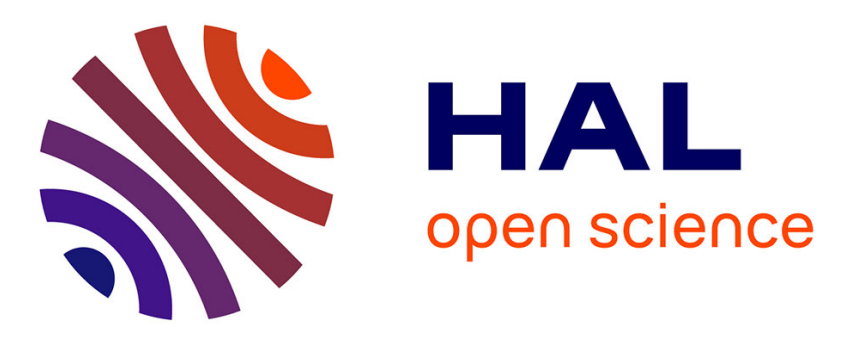

\title{
A phosphatase cascade by which rewarding stimuli control nucleosomal response
}

Alexandre Stipanovich, Emmanuel Valjent, Miriam Matamales, Akinori Nishi, Jung-Hyuck Ahn, Matthieu Maroteaux, Jesus Bertran-Gonzalez, Karen Brami-Cherrier, Hervé Enslen, Anne-Gaëlle Corbillé, et al.

\section{To cite this version:}

Alexandre Stipanovich, Emmanuel Valjent, Miriam Matamales, Akinori Nishi, Jung-Hyuck Ahn, et al.. A phosphatase cascade by which rewarding stimuli control nucleosomal response. Nature, 2008, 453 (7197), pp.879. 10.1038/nature06994 . cea-00944345

\section{HAL Id: cea-00944345 \\ https://hal-cea.archives-ouvertes.fr/cea-00944345}

Submitted on 24 Sep 2019

HAL is a multi-disciplinary open access archive for the deposit and dissemination of scientific research documents, whether they are published or not. The documents may come from teaching and research institutions in France or abroad, or from public or private research centers.
L'archive ouverte pluridisciplinaire HAL, est destinée au dépôt et à la diffusion de documents scientifiques de niveau recherche, publiés ou non, émanant des établissements d'enseignement et de recherche français ou étrangers, des laboratoires publics ou privés. 


\title{
ARTICLES
}

\section{A phosphatase cascade by which rewarding stimuli control nucleosomal response}

\author{
Alexandre Stipanovich ${ }^{1,2,3 *}$, Emmanuel Valjent ${ }^{1,2,3 *}$, Miriam Matamales ${ }^{1,2,3 *}$, Akinori Nishi ${ }^{4,5}$, Jung-Hyuck Ahn ${ }^{5}$, \\ Matthieu Maroteaux ${ }^{1,2,3}$, Jesus Bertran-Gonzalez ${ }^{1,2,3}$, Karen Brami-Cherrier ${ }^{1,2,3}$, Hervé Enslen ${ }^{1,2,3}$, \\ Anne-Gaëlle Corbillé ${ }^{1,2,3}$, Odile Filhol ${ }^{6}$, Angus C. Nairn ${ }^{5,7}$, Paul Greengard ${ }^{5}$, Denis Hervé ${ }^{1,2,3}$ \\ \& Jean-Antoine Girault ${ }^{1,2,3}$
}

Dopamine orchestrates motor behaviour and reward-driven learning. Perturbations of dopamine signalling have been implicated in several neurological and psychiatric disorders, and in drug addiction. The actions of dopamine are mediated in part by the regulation of gene expression in the striatum, through mechanisms that are not fully understood. Here we show that drugs of abuse, as well as food reinforcement learning, promote the nuclear accumulation of 32-kDa dopamine-regulated and cyclic-AMP-regulated phosphoprotein (DARPP-32). This accumulation is mediated through a signalling cascade involving dopamine D1 receptors, cAMP-dependent activation of protein phosphatase-2A, dephosphorylation of DARPP-32 at Ser 97 and inhibition of its nuclear export. The nuclear accumulation of DARPP-32, a potent inhibitor of protein phosphatase-1, increases the phosphorylation of histone $H 3$, an important component of nucleosomal response. Mutation of Ser 97 profoundly alters behavioural effects of drugs of abuse and decreases motivation for food, underlining the functional importance of this signalling cascade.

Midbrain dopamine neurons, activated after unexpected rewarding stimuli, are essential in reinforcement learning ${ }^{1}$. Drugs of abuse mimic the physiological action of dopamine neurons by increasing their firing rate or preventing the uptake of dopamine. Thus, they enhance extracellular dopamine levels in the forebrain, especially in the nucleus accumbens (NAc), a key structure required for the reinforcing effects of addictive drugs ${ }^{2-4}$. To understand how dopamine mediates natural or pathological reinforcement learning, it is necessary to identify the intracellular events that trigger gene transcription alterations supporting long-lasting synaptic changes ${ }^{5-7}$. DARPP-32 (ref. 8) is a prominent mediator of dopamine signalling in the striatum $^{9}$ and is highly enriched in striatal medium-size spiny GABA neurons ${ }^{10}$. After activation of the dopamine D1 receptor (D1R), DARPP-32 is phosphorylated by cAMP-dependent protein kinase (PKA) at Thr 34 and converted into a potent inhibitor of the multifunctional serine/threonine protein phosphatase-1 (PP1) ${ }^{11}$. DARPP32-mediated inhibition of PP1 increases the phosphorylation of neurotransmitter receptors and ion channels crucial for synaptic function and plasticity ${ }^{9}$. DARPP-32 also regulates nuclear events, as demonstrated by alterations of drug-induced gene expression in mice lacking DARPP-32 or bearing a point mutation of Thr 34 (refs 12, 13). Part of the control exerted by DARPP-32 on transcription is mediated by activation of the extracellular signal-regulated kinase pathway, which is dependent on the concomitant stimulation of D1R and glutamate $N$-methyl-D-aspartate receptors ${ }^{13,14}$. However, the precise mechanisms of information transfer from the cytoplasm to the nucleus of striatal neurons are still poorly understood.

\section{Drugs and incentive learning increase nuclear DARPP-32}

DARPP-32 has been extensively characterized as a cytoplasmic protein ${ }^{9}$. In contrast, electron microscopy has revealed immunoreactivity to DARPP-32 in the nucleus of some striatal medium-size spiny neurons ${ }^{15}$, although the significance of this observation has not been explained. In mice treated with D-amphetamine, immunoreactivity to DARPP-32 phosphorylated on Thr 34 was strong in the nuclei of striatal neurons (Fig. 1a). D-Amphetamine also increased total DARPP-32 immunoreactivity in nuclei (Fig. 1a, b). Cocaine induced a rapid accumulation of DARPP-32 in the nucleus that lasted several hours (Fig. 1b and Supplementary Fig. 1a). D-Amphetamine and cocaine triggered the nuclear accumulation of DARPP-32 in both the dorsal striatum and the NAc (data not shown). In contrast, morphine increased nuclear DARPP-32 only in the NAc shell (Fig. 1b and Supplementary Fig. 1b), where it potently increases dopamine release ${ }^{16}$. A reinforcement learning model, in which mice nose-poked for food, also triggered the nuclear accumulation of DARPP-32 in the dorsal striatum and in the NAc shell and core (Fig. 1c). In mice receiving no food, or in yoked mice that received food when the 'active' animal nose-poked, no nuclear accumulation was observed (Fig. 1c).

In drd1a-EGFP mice, which specifically express enhanced green fluorescent protein (EGFP) in striatonigral neurons under the control of the D1R promoter $(d r d 1 a)^{17}$, nuclear accumulation of DARPP-32 occurred in D1R-expressing neurons $8 \mathrm{~min}$ after cocaine injection (Supplementary Fig. 2a). Cocaine-induced rapid nuclear accumulation of DARPP-32 was not observed in D1R-null mice (Supplementary Fig. 2b), indicating the requirement for D1R. In striatal neurons in culture, DARPP-32 immunofluorescence was predominantly cytoplasmic; application of SKF81297, a D1R agonist, induced its nuclear accumulation, which was prevented by a D1R antagonist, SCH23390, or a cAMP antagonist, Rp-cAMPS (Supplementary Fig. 3a, b). Conversely, a cAMP analogue, Sp-5,6-DClcBIMPS, increased nuclear DARPP-32 immunoreactivity in a 
time-dependent manner (Supplementary Fig. 3c). Similar results were obtained with DARPP-32 fused to EGFP (D32-GFP), coexpressed with D1R in striatal neurons in culture (Supplementary Fig. $4 a-c)$. Taken together, our results show that nuclear accumulation of DARPP-32 in response to physiological reward-controlled learning or to drugs of abuse was mediated by D1R and that CAMP was necessary and sufficient for this effect.

\section{Ser 97 phosphorylation controls DARPP-32 localization}

Leptomycin B (LMB), a specific inhibitor of CRM1-mediated nuclear export ${ }^{18}$, induced a rapid nuclear accumulation of endogenous DARPP-32 (Fig. 2a) or transfected D32-GFP (Supplementary Fig. 5a and Supplementary Video) in virtually all neurons. At residues 103111 of DARPP-32 we identified a putative nuclear export signal (NES; Fig. 2b) that was homologous to sequences known to bind CRM1 (ref. 19). Mutation of Leu 103 or Leu 109 to alanine induced a marked accumulation of D32-GFP in the nucleus (Fig. 2c), confirming the critical role of this sequence in nuclear export. Similar results were observed in Chinese hamster ovary cells (Supplementary Fig. 5b, c). We also found that the amino-terminal region of DARPP32, which encompasses several putative nuclear localization signals, had a critical function in its nuclear import (data not shown).
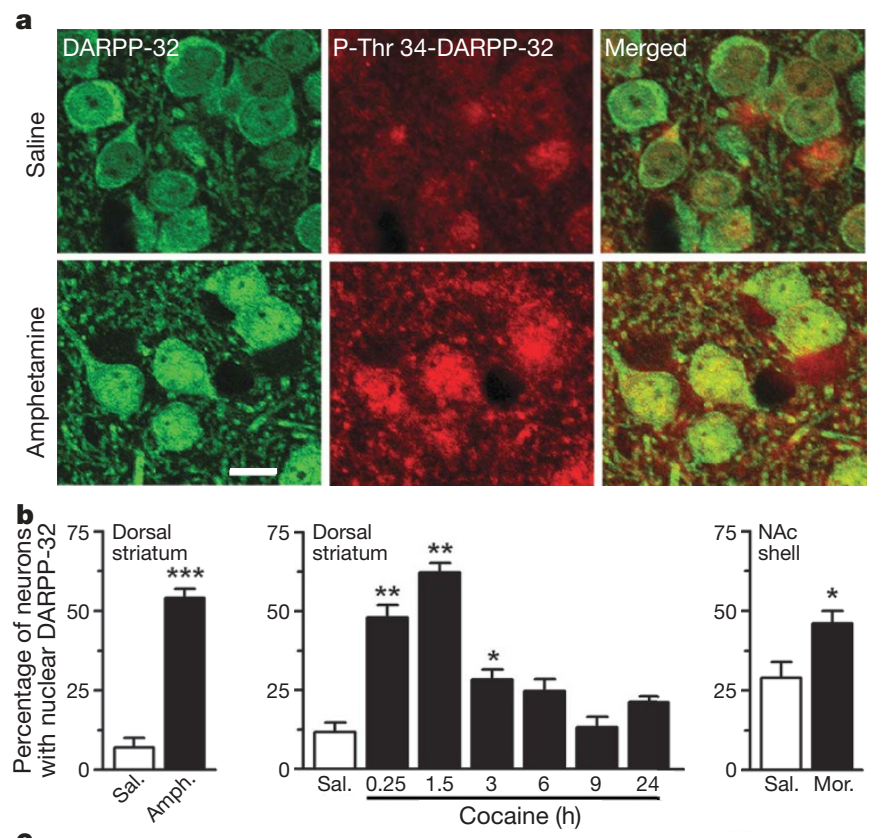

C
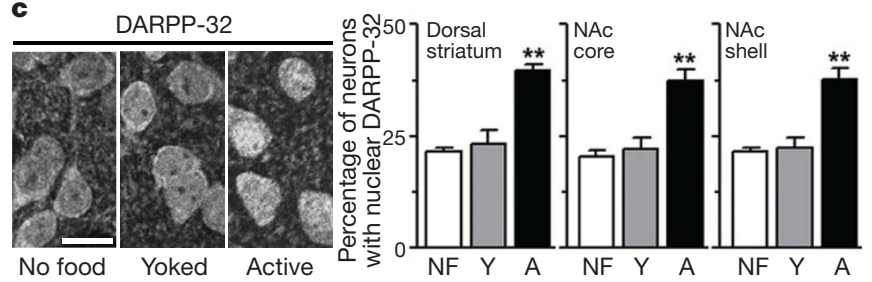

Figure 1 | Drugs of abuse and food self-administration induce nuclear accumulation of DARPP-32 in striatal neurons. a, DARPP-32 (green) and phospho-Thr 34-DARPP-32 (red) immunofluorescence in dorsal striatum of mice injected with saline or D-amphetamine $\left(10 \mathrm{mg} \mathrm{kg}^{-1}\right.$ intraperitoneally for $15 \mathrm{~min})$. b, Effects of D-amphetamine (amph.), cocaine $\left(20 \mathrm{mg} \mathrm{kg}^{-1}\right.$, time course) and morphine (mor., $5 \mathrm{mg} \mathrm{kg}^{-1}$ subcutaneously). Sal, saline. c, Effects of $1 \mathrm{~h}$ of reinforcement learning: mice in self-administration chamber without food (NF), learning to nose-poke for food pellets (active; A) or receiving pellets when the active animal nose-poked (yoked; Y). Error bars represent s.e.m., $n=4-8, t$-test (amphetamine, morphine) or one-way analysis of variance (Supplementary Table 1) and Bonferroni test. Asterisk, $P<0.05$; two asterisks, $P<0.01$; three asterisks, $P<0.001$. Single confocal sections. Scale bars, $10 \mu \mathrm{m}$.
These results suggested that D1R stimulation acted by reducing the export of DARPP-32 out of the nucleus. We ruled out a general inhibitory influence of D1R stimulation on CRM1-dependent nuclear export, by showing that SKF81297 did not alter the localization of another protein with an active $\mathrm{NES}^{20}$, namely $\mathrm{Ca}^{2+} /$ calmodulindependent kinase-I $\alpha$ (CaMKI $\alpha-$ GFP; Fig. 2d). Introduction of an ectopic NES sequence derived from prohibitin ${ }^{21}\left(\mathrm{NES}^{\mathrm{proh}}\right)$ at the

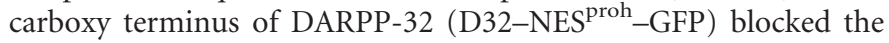
nuclear accumulation induced by SKF81297 (Fig. 2d).

To determine how the D1R/cAMP pathway regulated DARPP-32 localization we mutated its major phosphorylation sites (Thr 34, Thr 75, Ser 97 and Ser 130; Fig. 2b) to alanine. In striatal neurons in culture, basal and D1R-induced localization of T34A-D32-GFP, T75A-D32-GFP and S130A-D32-GFP mutants was similar to that of wild-type protein (Supplementary Fig. 6a). In support of these observations, in knock-in mutant mice bearing an alanine point mutation at Thr 34 or Thr 75, the basal localization of DARPP-32 and its cocaine-induced nuclear accumulation was similar to that of the wild type (Supplementary Fig. 6b, c). In contrast, when Ser 97, located close to the NES of DARPP-32, was mutated to alanine, the protein was nuclear in basal conditions and D1R stimulation did not alter this distribution (S97A-D32-GFP; Fig. 3a). Mutation of Ser 97 to the acidic residues glutamate (S97E) or aspartate (S97D), which mimics phosphorylation, induced a preferential cytoplasmic localization, which was not modified by SKF81297 (Fig. 3a and Supplementary Fig. 7a).

Ser 97 (Ser 102 in rat) is highly phosphorylated in basal conditions by casein kinase $2(\mathrm{CK} 2)^{22}$. An inhibitor of CK2, 4,5,6,7-tetrabromobenzotriazole (TBB $)^{23}$, markedly increased nuclear D32-GFP and prevented the effects of treatment with D1R agonist (Fig. 3b and Supplementary Fig. 7b). The effects of TBB resulted from the prevention of Ser 97 phosphorylation, because they were

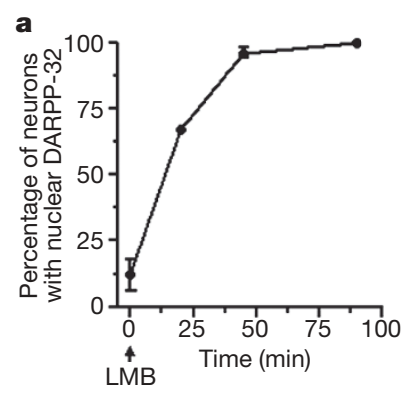

b
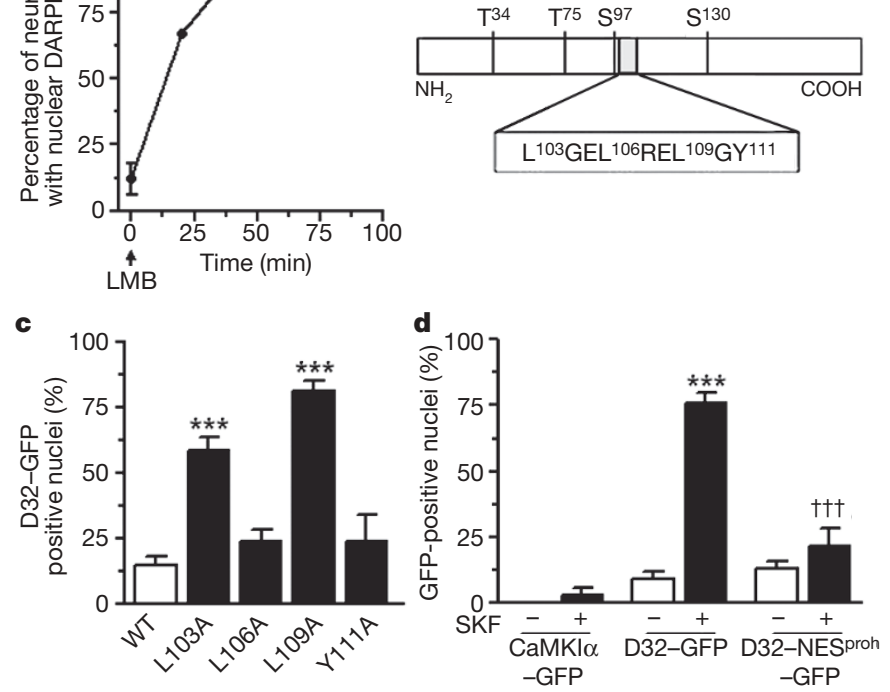

Figure 2 | DARPP-32 undergoes continuous cyto-nuclear shuttling. a, Leptomycin B (LMB; $10 \mathrm{ng} \mathrm{ml}^{-1}$ ) induces nuclear accumulation of DARPP-32 in striatal neurons in culture. $\mathbf{b}$, Putative NES in DARPP-32 and major phosphorylation sites. c, Mutagenesis of hydrophobic residues in NES increases nuclear localization of D32-GFP in transfected neurons. Three asterisks, $P<0.001$ compared with wild type (WT). d, Stimulation of D1R ( $10 \mu \mathrm{M}$ SKF81297 for $15 \mathrm{~min}$ ) has no effect on the localization of an unrelated protein with a NES (CaMKI $\alpha-$ GFP) or when DARPP-32 contains an additional NES (D32-NES ${ }^{\text {roh }}-$ GFP). Three asterisks, $P<0.001$ for SKF compared with vehicle; three daggers, $P<0.001$ for mutant compared with WT. Error bars represent s.e.m., $n=3-6$, one-way analysis of variance (Supplementary Table 1), Bonferroni test. 
not observed with S97E-D32-GFP (Fig. 3c and Supplementary Fig. 7c). These results support the hypothesis that DARPP-32 is mainly nuclear when Ser 97 is dephosphorylated, whereas it is preferentially cytoplasmic when phosphorylated by CK2.

\section{Protein phosphatase-2A dephosphorylates Ser 97}

Because our results predicted that dephosphorylation of Ser 97 would enhance nuclear DARPP-32, we tested the role of protein phosphatase-2A (PP2A), which dephosphorylates Ser 97 in vitro ${ }^{22}$. Pretreatment of striatal neurons with okadaic acid, a potent inhibitor of PP2A, did not alter the basal cytoplasmic localization of D32-GFP, whereas it blocked its D1R-induced nuclear translocation (Fig. 3d and Supplementary Fig. 7d). We next examined the regulation of Ser 97 phosphorylation in striatal slices ${ }^{24}$. SKF81297 induced a marked increase in Thr 34 phosphorylation, as expected, and a dephosphorylation of Ser 97 (Fig. 3e). The precise time course of the two responses was different, with dephosphorylation of Ser 97 being slower and more persistent than Thr 34 phosphorylation. Forskolin, a potent activator of adenylyl cyclase, induced a pronounced and sustained increase in Thr 34 phosphorylation and a slightly delayed, but persistent, decrease in Ser 97 phosphorylation (Fig. 3f and Supplementary Fig. 8). Forskolin-stimulated dephosphorylation of Ser 97 was prevented by okadaic acid (Fig. $3 \mathrm{~g}$ ), at a concentration that blocks PP2A activity fully, and PP1 activity only partly, in striatal slices ${ }^{25}$.

PP2A activity is controlled by various regulatory subunits, among which the B56 $\delta$ subunit is highly expressed in the striatum (Supplementary Fig. 9a) and is activated by cAMP-dependent phosphorylation $^{26,27}$. When B56 $\delta$ was co-expressed with DARPP-32 in
HEK293 cells, it promoted dephosphorylation of Ser 97 in response to forskolin (Fig. 3h and Supplementary Fig. 9b). In contrast, in cells transfected with the vector alone or with another isoform of the B subunit $(B \alpha)$, forskolin slightly increased Ser 97 phosphorylation (Fig. 3h and Supplementary Fig. 9b). Thus, D1R stimulation and increased cAMP levels induce the dephosphorylation of Ser 97 selectively through stimulation of PP2A containing the B56 $\delta$ subunit.

\section{Altered behaviour in S97A-DARPP-32 mice}

To examine the role of Ser 97 in vivo, we used a knock-in mouse line bearing a point mutation of this residue to alanine (S97A). DARPP32 was nuclear in about half of medium-size spiny neurons in the various striatal regions of the mutant mice (Fig. 4a). Cocaine injection did not alter the nuclear localization of S97A-DARPP-32 (Fig. 4a). The spontaneous activity, habituation and acute locomotor response to cocaine were similar in S97A-DARPP-32 mice and wildtype mice (Supplementary Fig. 10a-c), whereas the acute locomotor response to morphine $\left(5 \mathrm{mg} \mathrm{kg}^{-1}\right)$ was decreased in the mutant (Supplementary Fig. 10d). In wild-type mice a second injection of cocaine or morphine, seven days after the first injection, had a much stronger effect on locomotion than the first, showing a robust sensitization (Supplementary Fig. 10e, f) as reported previously ${ }^{13}$. In S97A-DARPP-32 mice, locomotor sensitization to cocaine and morphine, although still present, was less pronounced than in wild-type mice (Fig. 4b and Supplementary Fig. 10e, f). Moreover, the rewarding effects of cocaine, evaluated by conditioned place preference, were not observed in S97A-DARPP-32 mice (Fig. 4c).

We next examined whether mutation of Ser 97 interfered with responses to physiological rewarding stimuli by using a model in
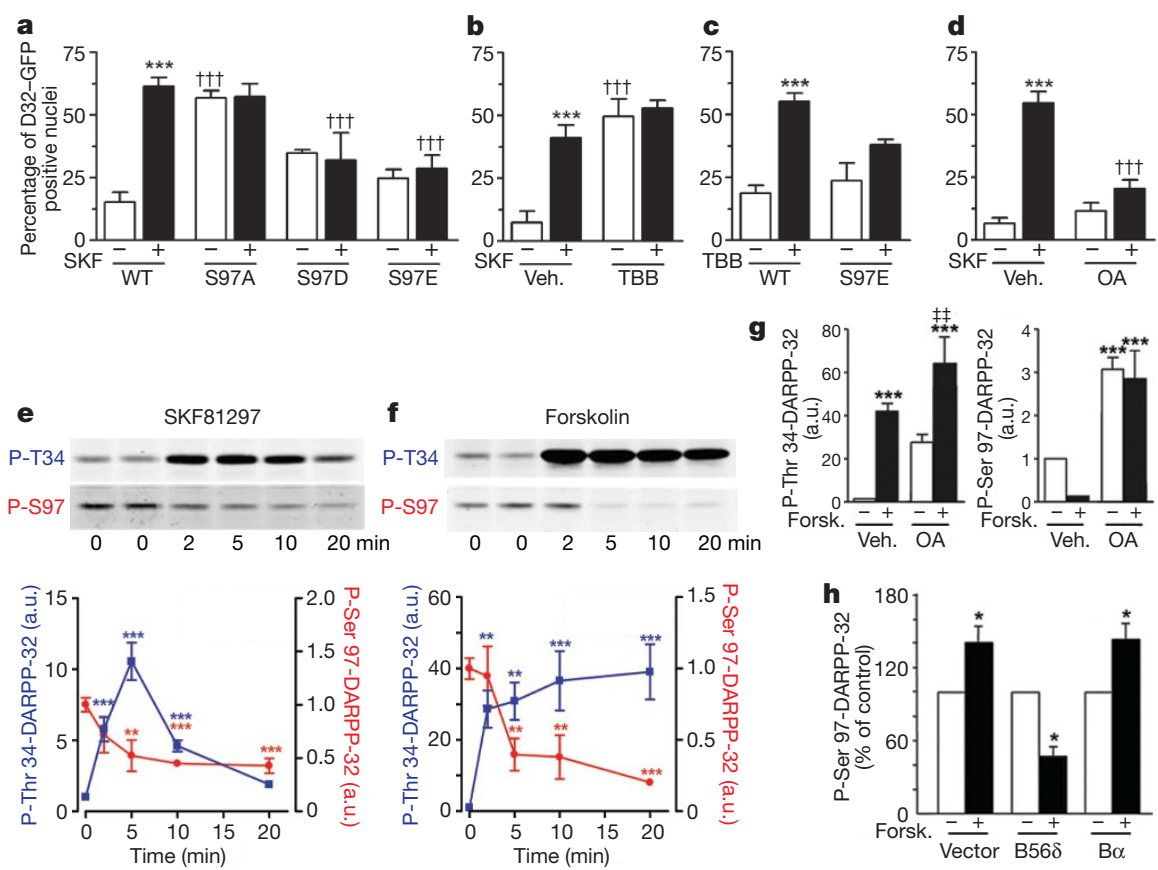

Figure 3 | Phosphorylation at Ser 97 controls intracellular localization of DARPP-32. a, Striatal neurons transfected with D1R and wild-type (WT), S97A-D32-GFP, S97D-D32-GFP or S97E-D32-GFP, treated with vehicle (Veh., -) or $10 \mu \mathrm{M}$ SKF81297 (SKF,,$+ 15 \mathrm{~min}$ ). Three asterisks, $P<0.001$ for SKF compared with vehicle; two daggers, $P<0.01$; three daggers, $P<0.001$ for mutant compared with WT. b, CK2 inhibition (TBB, $50 \mu \mathrm{M}$, $45 \mathrm{~min}$ before SKF) increases nuclear DARPP-32. Three asterisks, $P<0.001$ for SKF compared with vehicle; three daggers, $P<0.001$ for TBB compared with vehicle. c, S97E mutation prevents TBB effects. Three asterisks, $P<0.001$ for TBB compared with vehicle. d, Okadaic acid $(\mathrm{OA} ; 500 \mathrm{nM}$, 45 min before SKF) prevents D1R-induced DARPP-32 nuclear accumulation. Three asterisks, $P<0.001$ for SKF compared with vehicle; three daggers, $P<0.001$ for OA compared with vehicle. e, SKF81297
$(10 \mu \mathrm{M})$ induces Thr 34 phosphorylation (blue) and Ser 97 dephosphorylation (red) in mouse striatal slices (immunoblotting; $n=5-7)$. Two asterisks, $P<0.01$; three asterisks, $P<0.001$. f, Forskolin $(10 \mu \mathrm{M})$ also induces Thr 34 phosphorylation (blue) and Ser 97 dephosphorylation (red) ( $n=5-7)$. Two asterisks, $P<0.01$; three asterisks, $P<0.001$. g, Okadaic acid $(1 \mu \mathrm{M})$ prevents this forskolin-induced Ser 97 dephosphorylation. Three asterisks, $P<0.001$ compared with control; two double daggers, $P<0.01$ compared with OA. h, B56 $\delta$ allows Ser 97 dephosphorylation by forskolin (Forsk., $10 \mu \mathrm{M}, 10 \mathrm{~min}$ ): HEK293 cells transfected with DARPP-32 and vector, B56 $\delta$-PP2A or B $\alpha$-PP2A subunit (immunoblotting, $n=3$, $t$-test). Error bars represent s.e.m., one-way ANOVA (Supplementary Table 1) and Bonferroni test (unless otherwise indicated). Asterisk, $P<0.05$. 
which mildly food-deprived mice learned to obtain food pellets by nose-poking. Wild-type and mutant mice learned equally well, even when the number of nose-pokes necessary to obtain a pellet was increased to a fixed ratio of five or when the rewarded hole was reversed (Supplementary Fig. 10g). However, when the required number of nose-pokes was progressively increased until the mice interrupted their behaviour, the breaking point was lower in S97A mutant mice than in wild-type mice (Fig. 4d). Taken together, these results show that mutation of Ser 97 alters long-lasting responses to drugs of abuse, and decreases motivation for food reward.

In S97A-DARPP-32 mice we found a decreased phosphorylation of DARPP-32 on Thr 34 and of several proteins regulated through DARPP-32 (data not shown). We previously observed, with purified bovine DARPP-32, that phosphorylation by CK2 facilitated the phosphorylation of Thr 34 by PKA $^{22}$. However, there was no deficit in cAMP-induced Thr 34 phosphorylation of S97A mutant protein in transfected COS7 cells in which overexpressed DARPP-32 was cytoplasmic (Supplementary Fig. 11). We therefore conclude that the observed alterations in S97A mice probably resulted from the blockade of cyto-nuclear shuttling of DARPP-32, rather than from an intrinsic effect of Ser 97 phosphorylation on Thr 34 phosphorylation.

\section{Nuclear DARPP-32 regulates histone H3 phosphorylation}

Because D1R stimulation induces the accumulation of Thr34phospho-DARPP-32 (Fig. 1a), a potent inhibitor of PP1, we proposed that DARPP-32 translocation might regulate the phosphorylation of nuclear proteins. We therefore investigated the phosphorylation of histone $\mathrm{H} 3$ on Ser 10, a substrate for several kinases $^{28,29}$, which is known to be regulated by PP1 (refs 30, 31). The phosphorylation of $\mathrm{H} 3$ on Ser 10 is a key step in nucleosomal
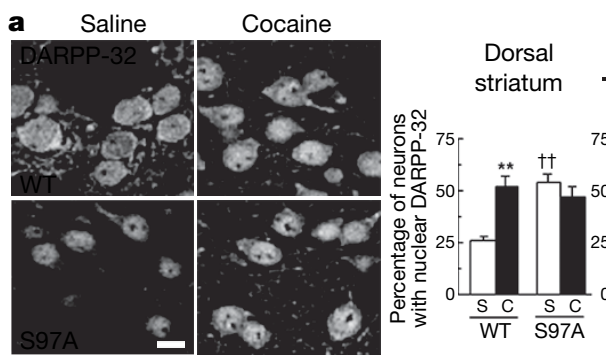

Accumbens

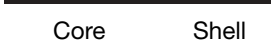

b
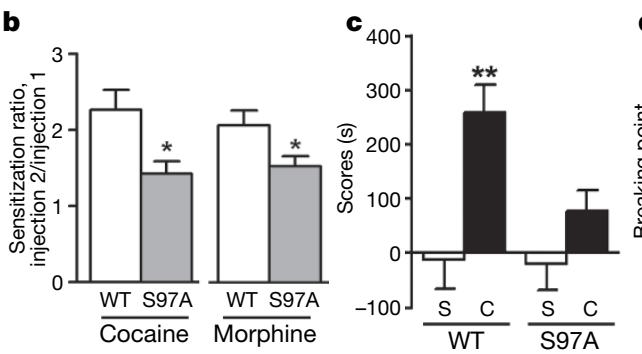

d

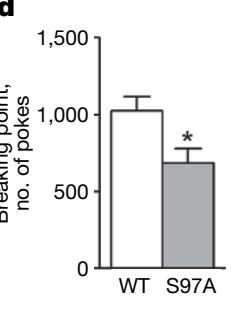

Figure 4 | Mutation of Ser 97 alters localization of DARPP-32, behavioural responses to drugs of abuse and motivation for food reward. a, DARPP-32 immunoreactivity (dorsal striatum) in wild-type (WT) or S97A-DARPP-32 mice injected with saline $(S)$ or cocaine $\left(C, 20 \mathrm{mg} \mathrm{kg}^{-1}\right.$, for $\left.10 \mathrm{~min}\right)$. Single confocal sections. Scale bar, $10 \mu \mathrm{m} ; n=3-8$. Asterisk, $P<0.05$; two asterisks, $P<0.01$ for saline compared with cocaine; dagger, $P<0.05$; two daggers, $P<0.01$ for S97A compared with WT. b, Decreased locomotor sensitization to a second injection (day 7) of cocaine $\left(20 \mathrm{mg} \mathrm{kg}^{-1}\right.$, for $1 \mathrm{~h}$; $n=16$ ) or morphine ( $5 \mathrm{mg} \mathrm{kg}^{-1}$, for $\left.3 \mathrm{~h} ; n=8\right)$ in S97A mice ( $t$-test).

Asterisk, $P<0.05$. c, Conditioned place preference to cocaine was prevented in S97A mice (scores are calculated as time in cocaine-paired compartment after conditioning minus that before conditioning; $n=7-8$ ). Two asterisks, $P<0.01$. d, Decreased breaking point for food reward under progressive ratio schedule in S97A mice ( $t$-test, $n=7-8$ ). Error bars represent s.e.m., two-way analysis of variance (Supplementary Table 1) and Bonferroni test (unless otherwise indicated). Asterisk, $P<0.05$. response $\mathrm{e}^{28,32}$ and is crucial for memory formation ${ }^{33}$. It is increased in the striatum after cocaine administration in vivo, and this response is functionally important ${ }^{14,34}$. Cocaine induced an intense phosphoSer 10-H3 signal in the nuclei of several striatal neurons in wild-type mice, whereas this effect was absent in both T34A-DARPP-32 and S97A-DARPP-32 mice (Fig. 5a). Similar results were observed with an antibody that detected phospho-acetyl-H3 (phospho-Ser 10acetyl-Lys 14-H3; data not shown).

In striatal neurons transfected with D1R and D32-GFP, the D1R agonist SKF81297 markedly increased the phosphorylation of $\mathrm{H} 3$ on Ser 10 (Fig. 5b, c, and Supplementary Fig. 12). SKF81297 did not alter the acetylation of $\mathrm{H} 3$ on Lys 14, but increased the immunoreactivity of phospho-Ser 10, acetyl-Lys 14 H3 (Fig. 5b and Supplementary Fig. 12). D1R-induced phosphorylation of histone H3 was lost when neurons were transfected with T34A-D32-GFP instead of the wild type (Fig. 5c), confirming that the ability of DARPP-32 to inhibit PP1 was essential for $\mathrm{H} 3$ phosphorylation. We then directly evaluated the role of DARPP-32 translocation to the nucleus by using mutant forms of D32-GFP that are constitutively excluded from the nucleus:

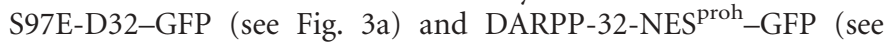
Fig. 2d). In neurons transfected with these 'cytoplasmic' variants of D32-GFP, the D1R agonist SKF81297 did not induce H3 phosphorylation (S97E and NES ${ }^{\text {proh }}$; Fig. 5c). These results showed that DARPP-32 phosphorylation on Thr 34 , and thus PP1 inhibition, was essential for D1R-induced phosphorylation of Ser 10-H3 and that this effect required the ability of DARPP-32 to accumulate in the nucleus in response to stimulation of D1R.

\section{Discussion}

Our study identifies nuclear DARPP-32 as a key factor in the phosphorylation of histone $\mathrm{H} 3$, a component of the nucleosomal response that is essential for gene expression ${ }^{28,32,33}$. Psychostimulant drugs (Damphetamine and cocaine) and morphine, which act by completely different mechanisms, share the ability to increase extracellular dopamine in the NAc and to trigger the nuclear accumulation of DARPP32. A natural stimulus such as a simple food-reinforced learning model caused a similar effect. DARPP-32 undergoes a very active, continuous cyto-nuclear shuttling regulated by the phosphorylation of Ser 97, which is in the vicinity of its NES (summarized in Fig. 5d). Phosphorylation of Ser 97 by CK2 seems to be crucial for the nuclear export of DARPP-32. CK2 is present in nuclei of striatal neurons (Supplementary Fig. 13) and can phosphorylate nuclear DARPP-32 and promote its export. Stimulation of D1R triggers the rapid phosphorylation of Thr 34 responsible for cytoplasmic effects of DARPP-32, and the slower dephosphorylation of Ser 97, by activating PP2A through cAMP/PKA-mediated phosphorylation of its B56 $\delta$ subunit ${ }^{26,27}$, thus decreasing the nuclear export of DARPP-32. Although phosphorylation has been reported to regulate the cytonuclear shuttling of several proteins $s^{35}$, this is a rare example of facilitation of CRM1-mediated nuclear export by phosphorylation $^{36,37}$. cAMP-independent mechanisms also couple dopamine $\mathrm{D} 2$ receptor (D2R) to a delayed activation of PP2A (ref. 38). D2R does not seem to be important in the early nuclear accumulation of DARPP-32, but it could have a regulatory role on Ser 97 phosphorylation and DARPP-32 localization in other circumstances.

A large body of evidence demonstrates that long-term synaptic plasticity requires the control of gene expression in the striatum as in other brain regions ${ }^{39}$. We have identified a previously unrecognized mechanism by which dopamine controls chromatin through the regulated translocation of a PP1 inhibitor to the nucleus, which promotes histone $\mathrm{H} 3$ phosphorylation. Dopamine-controlled inhibition of nuclear PP1, a wide-spectrum protein phosphatase, is likely to have other targets, making it a general means for the control of nuclear function. This mechanism is undoubtedly important in the long-term effects of drugs of abuse, which activate gene transcription through the stimulation of D1R, and in physiological rewardcontrolled learning. Regulation of the nuclear accumulation of 
a
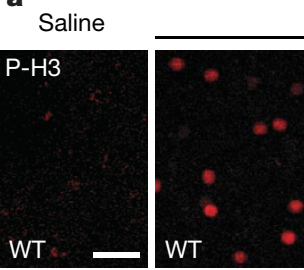

Cocaine
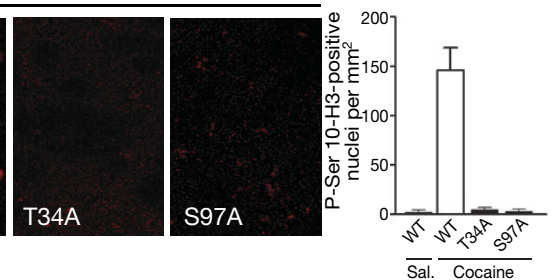

b

Vehicle
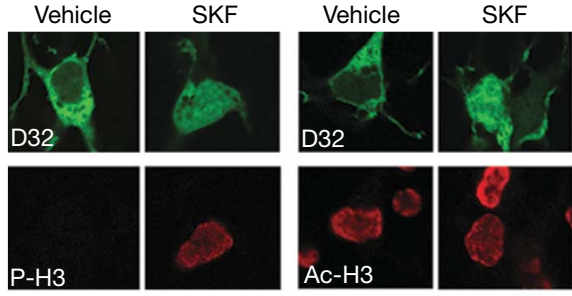

Vehicle

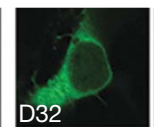

SKF
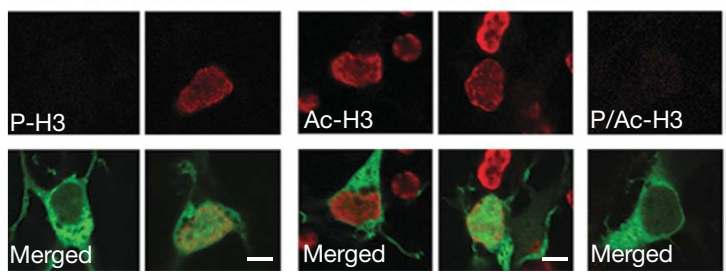

c

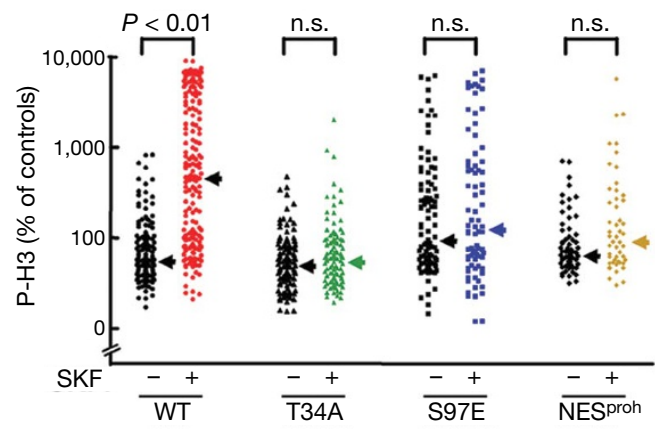

d

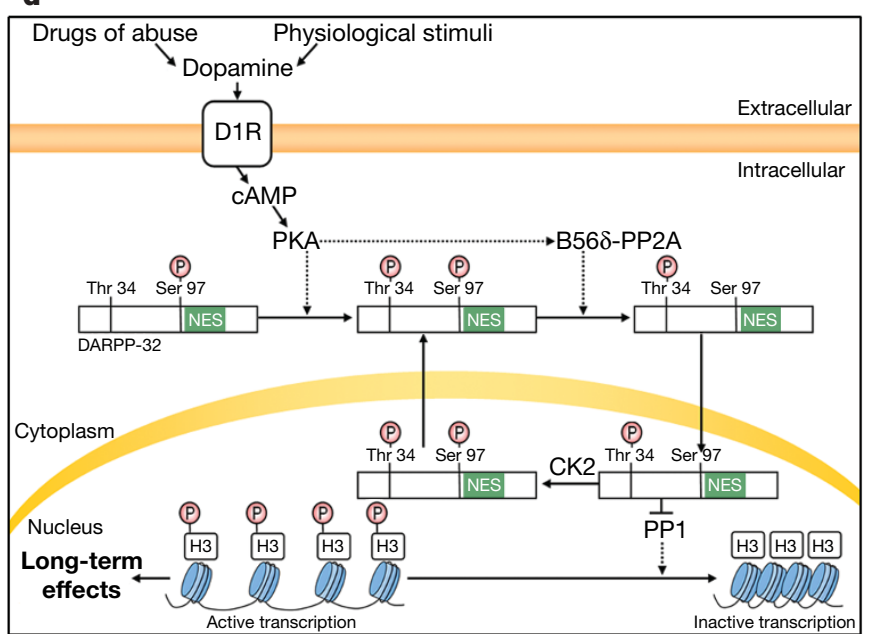

Figure 5 | Role of nuclear DARPP-32 in the phosphorylation of histone H3 in striatal neurons. a, Phospho-Ser 10-H3 (P-H3) immunofluorescence induced by cocaine $\left(20 \mathrm{mg} \mathrm{kg}^{-1}\right.$, for $\left.30 \mathrm{~min}\right)$ in wild-type (WT) dorsal striatum is abolished in T34A or S97A DARPP-32 mutant mice. Scale bar, $40 \mu \mathrm{m}$. b, Stimulation of D1R (SKF81297 (SKF), $10 \mu \mathrm{M}$, for $15 \mathrm{~min}$ ) increases Ser 10-H3 phosphorylation in striatal neurons transfected with D32-GFP and D1R. Confocal section, GFP fluorescence, P-H3, acetylLys 14-H3 (Ac-H3) and phospho-Ser 10-acetyl-Lys 14-H3 (P/Ac-H3) immunofluorescence. Scale bars, $5 \mu \mathrm{m}$. c, Phosphorylation of $\mathrm{H} 3$ requires DARPP-32 Thr 34 phosphorylation and nuclear accumulation. Striatal neurons transfected with WT, T34A-D32-GFP, S97E-D32-GFP or

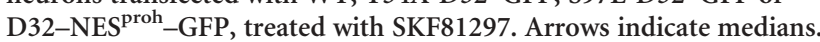
Kruskal-Wallis $(P<0.0001)$ and Dunn's tests. n.s., not significant. d, Working model of regulation of nucleosomal response in striatal neurons by DARPP-32.
DARPP-32 may also be important to the functions or dysfunctions of dopamine that involve transcription-dependent long-term plasticity, including neuroleptic- and L-DOPA-induced dyskinesia, and other diseases of the basal ganglia.

\section{METHODS SUMMARY}

Knock-in T34A-DARPP-32, T75A-DARPP-32 and S97A-DARPP-32 (refs 40, $41), \mathrm{D} 1 \mathrm{R}-$ deficient ${ }^{42}, d r d 1 a$-EGFP ${ }^{17}$ and matched wild-type control mice were habituated to saline injections for three days before experiments. Drug-treated mice were anaesthetized with pentobarbital and received an intracardiac perfusion of $4 \%$ paraformaldehyde. Vibratome-cut brain sections were processed for immunofluorescence ${ }^{13}$. Primary striatal neurons, from 14-day embryonic Swiss mice, were grown in supplemented Neurobasal medium and transfected a week later with plasmids expressing D1R and DARPP-32 fused with C-terminal EGFP (D32-GFP). HEK293, CHO-K1 or COS-7 cells were transfected with DARPP-32 constructs associated or not with PP2A subunits, and studied 24$48 \mathrm{~h}$ later. On the basis of confocal single sections, the subcellular localization of DARPP-32 or GFP was classified into three categories: nuclear staining stronger than, equal to or weaker than cytoplasmic. For cells in culture, the first two categories were pooled and considered as cells with nuclear staining. Phosphorylated and/or acetylated H3 immunofluorescence was quantified with Image-J-1.34s (National Institutes of Health). Striatal coronal slices $(350 \mu \mathrm{m})$ were incubated in oxygenated artificial cerebrospinal fluid ${ }^{25}$, and phosphoDARPP-32 was analysed in slices or transfected cells by western blotting ${ }^{25}$. Locomotor activity, sensitization produced by a single injection of drug, and conditioned place preference were measured as described ${ }^{13,14,43}$. In the operant model for food self-administration (incentive learning), mildly food-deprived mice were placed in an operant chamber with two nose-poke holes. One or five nose-pokes (fixed ratios 1 and 5) in the rewarded hole, indicated by a cue light, triggered the release of a food pellet. Controls were 'no food' mice (mice in the same chamber but with no pellet delivered) and 'yoked' mice (paired to active mice, in exactly the same conditions, but rewarded when the active mouse nosepoked). For the reversal test, the previously inactive hole became active and vice versa. For analysis of the progressive ratio, the number of successive pokes required for pellet release was progressively increased until the mouse stopped poking (breaking point).

Full Methods and any associated references are available in the online version of the paper at www.nature.com/nature.

Received 20 January; accepted 4 April 2008.

Published online 21 May 2008.

1. Schultz, W. \& Dickinson, A. Neuronal coding of prediction errors. Annu. Rev. Neurosci. 23, 473-500 (2000).

2. Berke, J. D. \& Hyman, S. E. Addiction, dopamine, and the molecular mechanisms of memory. Neuron 25, 515-532 (2000).

3. Di Chiara, G. Drug addiction as dopamine-dependent associative learning disorder. Eur. J. Pharmacol. 375, 13-30 (1999).

4. Everitt, B. J. \& Robbins, T. W. Neural systems of reinforcement for drug addiction: from actions to habits to compulsion. Nature Neurosci. 8, 1481-1489 (2005).

5. Nicola, S. M., Surmeier, J. \& Malenka, R. C. Dopaminergic modulation of neuronal excitability in the striatum and nucleus accumbens. Annu. Rev. Neurosci. 23, 185-215 (2000).

6. Reynolds, J. N. \& Wickens, J. R. Dopamine-dependent plasticity of corticostriatal synapses. Neural Netw. 15, 507-521 (2002)

7. Hyman, S. E., Malenka, R. C. \& Nestler, E. J. Neural mechanisms of addiction: the role of reward-related learning and memory. Annu. Rev. Neurosci. 29, 565-598 (2006).

8. Walaas, S. I., Aswad, D. W. \& Greengard, P. A dopamine- and cyclic AMPregulated phosphoprotein enriched in dopamine-innervated brain regions. Nature 301, 69-71 (1983).

9. Svenningsson, P. et al. DARPP-32: an integrator of neurotransmission. Annu. Rev. Pharmacol. Toxicol. 44, 269-296 (2004).

10. Ouimet, C. C. et al. DARPP-32, a dopamine- and adenosine $3^{\prime}: 5^{\prime}-$ monophosphate-regulated phosphoprotein enriched in dopamine-innervated brain regions. III. Immunocytochemical localization. J. Neurosci. 4, 111-124 (1984).

11. Hemmings, H. C. Jr, Greengard, P., Tung, H. Y. L. \& Cohen, P. DARPP-32, a dopamine-regulated neuronal phosphoprotein, is a potent inhibitor of protein phosphatase-1. Nature 310, 503-505 (1984).

12. Fienberg, A. A. et al. DARPP-32: Regulator of the efficacy of dopaminergic neurotransmission. Science 281, 838-839 (1998).

13. Valjent, E. et al. Regulation of a protein phosphatase cascade allows convergent dopamine and glutamate signals to activate ERK in the striatum. Proc. Natl Acad. Sci. USA 102, 491-496 (2005).

14. Brami-Cherrier, K. et al. Parsing molecular and behavioral effects of cocaine in mitogen- and stress-activated protein kinase-1-deficient mice. J. Neurosci. 25, 11444-11454 (2005) 
15. Ouimet, C. C. \& Greengard, P. Distribution of DARPP-32 in the basal ganglia: An electron microscopic study. J. Neurocytol. 19, 39-52 (1990).

16. Pontieri, F. E., Tanda, G. \& Di Chiara, G. Intravenous cocaine, morphine, and amphetamine preferentially increase extracellular dopamine in the 'shell' as compared with the 'core' of the rat nucleus accumbens. Proc. Natl Acad. Sci. USA 92, 12304-12308 (1995).

17. Gong, S. et al. A gene expression atlas of the central nervous system based on bacterial artificial chromosomes. Nature 425, 917-925 (2003).

18. Nishi, K. et al. Leptomycin B targets a regulatory cascade of crm1, a fission yeast nuclear protein, involved in control of higher order chromosome structure and gene expression. J. Biol. Chem. 269, 6320-6324 (1994).

19. Henderson, B. R. \& Eleftheriou, A. A comparison of the activity, sequence specificity, and CRM1-dependence of different nuclear export signals. Exp. Cell Res. 256, 213-224 (2000).

20. Stedman, D. R. et al. Cytoplasmic localization of calcium/calmodulin-dependent protein kinase $\mathrm{I}-\alpha$ depends on a nuclear export signal in its regulatory domain. FEBS Lett. 566, 275-280 (2004).

21. Rastogi, S., Joshi, B., Fusaro, G. \& Chellappan, S. Camptothecin induces nuclear export of prohibitin preferentially in transformed cells through a CRM-1dependent mechanism. J. Biol. Chem. 281, 2951-2959 (2006).

22. Girault, J. A. et al. Phosphorylation of DARPP-32, a dopamine- and CAMPregulated phosphoprotein, by casein kinase II. J. Biol. Chem. 264, 21748-21759 (1989).

23. Sarno, S. et al. Selectivity of 4,5,6,7-tetrabromobenzotriazole, an ATP sitedirected inhibitor of protein kinase CK2 ('casein kinase-2'). FEBS Lett. 496, 44-48 (2001).

24. Nishi, A. et al. Amplification of dopaminergic signaling by a positive feedback loop. Proc. Natl Acad. Sci. USA 97, 12840-12845 (2000).

25. Nishi, A., Snyder, G. L., Nairn, A. C.\& Greengard, P. Role of calcineurin and protein phosphatase-2A in the regulation of DARPP-32 dephosphorylation in neostriatal neurons. J. Neurochem. 72, 2015-2021 (1999).

26. Usui, H. et al. Activation of protein phosphatase $2 \mathrm{~A}$ by cAMP-dependent protein kinase-catalyzed phosphorylation of the $74-k D a B^{\prime \prime}(\delta)$ regulatory subunit in vitro and identification of the phosphorylation sites. FEBS Lett. 430, 312-316 (1998).

27. Ahn, J. H. et al. Protein kinase A activates protein phosphatase $2 \mathrm{~A}$ by

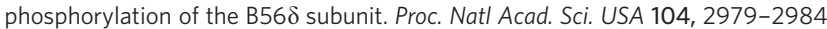
(2007).

28. Nowak, S. J. \& Corces, V. G. Phosphorylation of histone H3: a balancing act between chromosome condensation and transcriptional activation. Trends Genet. 20, 214-220 (2004).

29. Salvador, L. M. et al. Follicle-stimulating hormone stimulates protein kinase A-mediated histone $\mathrm{H} 3$ phosphorylation and acetylation leading to select gene activation in ovarian granulosa cells. J. Biol. Chem. 276, 40146-40155 (2001).

30. Murnion, M. E. et al. Chromatin-associated protein phosphatase 1 regulates aurora-B and histone H3 phosphorylation. J. Biol. Chem. 276, 26656-26665 (2001).

31. Hsu, J. Y. et al. Mitotic phosphorylation of histone $\mathrm{H} 3$ is governed by Ipl1/aurora kinase and Glc7/PP1 phosphatase in budding yeast and nematodes. Cell 102, 279-291 (2000).

32. Bode, A. M. \& Dong, Z. G. Inducible covalent postranslational modification of histone H3. Sci. STKE 281, 1-12 (2005).

33. Levenson, J. M. \& Sweatt, J. D. Epigenetic mechanisms: a common theme in vertebrate and invertebrate memory formation. Cell. Mol. Life Sci. 63, 1009-1016 (2006)

34. Kumar, A. et al. Chromatin remodeling is a key mechanism underlying cocaineinduced plasticity in striatum. Neuron 48, 303-314 (2005).
35. Poon, I. K. \& Jans, D. A. Regulation of nuclear transport: central role in development and transformation? Traffic 6, 173-186 (2005).

36. Panasyuk, G. et al. Nuclear export of $\mathrm{S} 6 \mathrm{~K} 1 \mathrm{II}$ is regulated by protein kinase CK2 phosphorylation at Ser-17. J. Biol. Chem. 281, 31188-31201 (2006).

37. Alt, J. R., Cleveland, J. L., Hannink, M. \& Diehl, J. A. Phosphorylation-dependent regulation of cyclin D1 nuclear export and cyclin D1-dependent cellular transformation. Genes Dev. 14, 3102-3114 (2000).

38. Beaulieu, J. M. et al. An Akt/ $\beta$-arrestin 2/PP2A signaling complex mediates dopaminergic neurotransmission and behavior. Cell 122, 261-273 (2005).

39. Nestler, E. J. Molecular basis of long-term plasticity underlying addiction. Nature Rev. Neurosci. 2, 119-128 (2001).

40. Svenningsson, P. et al. Diverse psychotomimetics act through a common signaling pathway. Science 302, 1412-1415 (2003).

41. Zhang, $Y$. et al. Cocaine self-administration in mice is inversely related to phosphorylation at Thr34 (protein kinase A site) and Ser130 (kinase CK1 site) of DARPP-32. J. Neurosci. 26, 2645-2651 (2006).

42. Drago, J. et al. Altered striatal function in a mutant mouse lacking D1A dopamine receptors. Proc. Natl Acad. Sci. USA 91, 12564-12568 (1994).

43. Vanderschuren, L. J. et al. A single exposure to amphetamine is sufficient to induce long-term behavioral, neuroendocrine, and neurochemical sensitization in rats. J. Neurosci. 19, 9579-9586 (1999).

44. Valjent, E. et al. Involvement of the extracellular signal-regulated kinase cascade for cocaine-rewarding properties. J. Neurosci. 20, 8701-8709 (2000).

Supplementary Information is linked to the online version of the paper at www.nature.com/nature.

Acknowledgements We thank M. Lambert for her help with time-lapse video; P. Ingrassia and P. Bernard for their help with mutant mice; and M. R. Picciotto, S. Cottecchia, J. P. Hornung, R. Luedtke, M. Takeda and Intracellular Therapies Inc. for reagents. This work was supported by Inserm, and by grants from Agence Nationale de la Recherche (05-NEUR-020-01), Fondation Bettencourt-Schueller (Coup d'élan) and Association pour la Recherche contre le Cancer (ARC-3118 and -7905) to J.A.G., from Fondation pour la Recherche Médicale (FRM) to D.H., a Grant-in-Aid for Scientific Research from the Japan Society for the Promotion of Science to A.N., and grants from the National Institute on Drug Abuse (DA10044), the National Institute of Mental Health (MH74866), the US Department of Defense (W81XWH-05-1-0146), the Picower Foundation, the Michael Stern Parkinson's Research Foundation and the US Army Medical Research Acquisition Activity (DAMD17-02-1-0705 and W81XWH-05-1-0146) to P.G. and A.C.N. A.S. was supported by Mission Interministérielle de Lutte contre la Drogue et la Toxicomanie and FRM, and J.B.G. by FRM.

Author Contributions A.S. and Mi.M. performed experiments in vivo and in transfected cultures, immunofluorescence and molecular biology. E.V. conducted in vivo, behavioural and immunohistofluorescence experiments. A.S., E.V. and Mi.M. prepared the figures. A.N. performed the slice experiments, J.H.A. the phosphatase experiments, and Ma.M. the incentive learning experiments. J.B.G. and A.G.C. contributed to in vivo and immunohistofluorescence experiments, and K.B.C. and H.E. to cell culture experiments. O.F. provided advice and reagents. A.S., A.C.N., P.G., D.H. and J.A.G. were involved in the study design and manuscript writing. J.A.G. coordinated the study. All authors analysed data they generated, discussed results and commented on the manuscript. A.S., E.V. and Mi.M. contributed equally to this work.

Author Information Reprints and permissions information is available at www.nature.com/reprints. Correspondence and requests for materials should be addressed to J-A.G. (girault@fer-a-moulin.inserm.fr). 


\section{METHODS}

Animals. Male eight-week-old C57BL/6J mice from Elevage Janvier or JapanSLC were used for in vivo and slice experiments, respectively. Production of knock-in T34A-DARPP-32, T75A-DARPP-32 and S97A-DARPP-32 mutant ${ }^{40,41}$, D1R-deficient $t^{42}$ and $d r d 1 a-\mathrm{EGFP}^{17}$ mice were described previously. Mutant and wild-type mice used in experiments, or their parents, were littermates. Mice were kept for at least one week in a local animal house in stable conditions $\left(22^{\circ} \mathrm{C}\right.$, $12 \mathrm{~h} / 12 \mathrm{~h}$ light/dark cycle, free access to food and water). During the three days preceding the experiments, mice were habituated to behavioural apparatus, when applicable, and to injections by daily intraperitoneal saline administration. Animal care was in accordance with ethical guidelines (Declaration of Helsinki and NIH, publication no. 85-23, revised 1985, European Community Guidelines, and French Agriculture and Forestry Ministry guidelines for handling animals, decree 87849, license A 75-05-22) and approved by the local ethical committees.

Drugs. (+)-D-Methylphenethylamine (D-amphetamine) sulphate salt, cocaine$\mathrm{HCl}$, SKF81297, SCH23390 (both in 0.1\% v/v dimethylsulphoxide) and okadaic acid potassium salt were from Sigma-Aldrich or Tocris; morphine sulphate salt was from Francopia; 5,6-dichloro-1- $\beta$-D-ribofuranosylbenzimidazole-3', $5^{\prime}$ cyclic-monophosphoro-thioate, Sp-isomer (Sp-5,6-DCl-cBIMPS) and adenosine$3^{\prime}, 5^{\prime}$-cyclic-mono-phosphorothioate, Rp-isomer (Rp-cAMPS) were from Biolog; leptomycin-B was from Amersham; 4,5,6,7-tetrabromobenzotriazole $(50 \mu \mathrm{M}$ in $0.1 \%$ dimethylsulphoxide) was from Calbiochem. For in vivo experiments, drugs were dissolved in $0.9 \% \mathrm{NaCl}$ and injected intraperitoneally (i.p.) except morphine, which was injected subcutaneously (s.c.).

Plasmid constructs and DARPP-32 mutants. Rattus norvegicus DARPP-32 cDNA (mouse residue numbering is used throughout) was amplified by PCR with Pfu (Stratagene) and cloned in the XhoI/EcoRI sites of EGFP-N2 (Clontech), and subjected to site-directed mutagenesis (Quick-Change; Stratagene). DARPP-32-NES ${ }^{\text {proh }}$ was constructed by EcoRI/SalI digestion and ligation of a sequence encoding AAEDIAYQLSRSRNITYLPAGQSVLLQLPQ (NES of prohibitin; ref. 21) to the C terminus of DARPP-32. Constructs were verified by DNA sequencing. CaMKI $\alpha-$ GFP and pRK5-D1R were gifts.

Cell culture and transfection. Striatal neurons (180,000 per well) from 14-day embryonic Swiss mice (Janvier) were transfected after seven days in culture ${ }^{14}$ with D32-GFP $(1 \mu \mathrm{g})$ and pRK5-D1R $(1 \mu \mathrm{g})$ DNA, using Lipofectamine 2000 in OptiMEM serum-free medium. Treatments were done $24 \mathrm{~h}$ later, in fresh Neurobasal medium. CHO-K1 cells (American Type Culture Collection) (25,000 cells per 14-mm diameter coverslip) were cultured in Ham's F-12 medium with $10 \%$ foetal bovine serum (FBS). COS-7 and HEK293 cells were cultured in six-well plates $\left(10^{6}\right.$ per well) in DMEM medium with $10 \%$ FBS. Culture media were from Invitrogen. $\mathrm{CHO}-\mathrm{K} 1$ and COS-7 cells were transfected as neurons with D32-GFP DNA $(1 \mu \mathrm{g})$. HEK293 cells were co-transfected with Myc-tagged DARPP-32 (0.5 $\mu \mathrm{g})$ and Flag-tagged B-subunits of PP2A vector, B $\alpha$ or B56 $\delta(0.5 \mu \mathrm{g})$ DNA, using Fugene-6 (Roche). After $48 \mathrm{~h}$, cells were treated with forskolin and lysed in $150 \mu \mathrm{l}$ of $50 \mathrm{mM}$ Tris- $\mathrm{HCl} \mathrm{pH} 7.4,150 \mathrm{mM} \mathrm{NaCl}, 1 \%$ Triton X-100, 0.1\% SDS, protease inhibitor cocktail (Roche) and phosphatase inhibitor cocktail I and II (Calbiochem), sonicated for $5 \mathrm{~s}$, and centrifuged for $10 \mathrm{~min}$ at $16,000 \mathrm{~g}$.

Immunostaining of striatal neurons in culture. Cells were fixed with PBS containing $2 \%$ paraformaldehyde $\left(40 \mathrm{~min}, 20-23{ }^{\circ} \mathrm{C}\right)$ and incubated with methanol/acetone $\left(1: 1,10 \mathrm{~min}, 4^{\circ} \mathrm{C}\right)$. After three rinses in PBS, cells were treated with blocking buffer (3\% BSA in PBS except for H3 immunofluorescence: 1\% BSA and $1 \% \mathrm{FBS}$ ) for $45 \mathrm{~min}$ ( $2 \mathrm{~h}$ for P-Ser $10-\mathrm{H} 3$ ) at room temperature. Coverslips were incubated overnight at $4{ }^{\circ} \mathrm{C}$ in PBS with $1 \%$ BSA and DARPP-32 (1:4,000 dilution) and D1R (1:500 dilution) monoclonal antibodies. P-Ser 10-H3, acetyl-Lys 14-H3 and P-Ser 10-acetyl-Lys 14-H3 antibodies (1:1,000 dilution; Upstate) were added overnight at $4{ }^{\circ} \mathrm{C}$ in PBS containing $1 \%$ BSA and $0.05 \%$ Tween 20. Anti-mouse Alexa488-conjugated antibody or anti-mouse Cy3-conjugated antibody (1:400 dilution; Molecular Probes) were added for $1 \mathrm{~h}$ at room temperature. Nuclei were counterstained with SYTOX (1:2,000 dilution; Molecular Probes). Cells rinsed three times were mounted under coverslips with Vectashield (Vector Laboratories).

Immunohistofluorescence of brain sections and subcellular distribution of DARPP-32. Brains from paraformaldehyde-perfused mice were postfixed and $30-\mu \mathrm{m}$ sections were cut with a vibratome (Leica) ${ }^{13,44}$. Free-floating sections were incubated overnight with antibodies at $4{ }^{\circ} \mathrm{C}$, and after rinses in TBS, for $2 \mathrm{~h}$ at room temperature with secondary antibody (1:400 dilution; Cy3-coupled anti-rabbit IgG or Alexa488-conjugated anti-mouse antibodies; Molecular Probes). Nuclei were counterstained with $1 \mu \mathrm{M}$ TO-PRO-3 iodide (Molecular Probes). Sections were rinsed three times in TBS and mounted in Vectashield. Image acquisition and statistical analysis. Image acquisition with sequential laser scanning confocal microscopy (SP2; Leica) was performed at the Institut du Fer à Moulin Imaging Facility. For brain immunofluorescence, two sections were analysed bilaterally and cells were counted in one field for dorsal striatum, NAc shell and core (data points for an animal correspond to four acquisitions per structure). For cultures, data points correspond to a minimum of 15 transfected cells (minimum of two different experiments with three independent transfections). Data were analysed with Prism (GraphPad) software, using parametric or non-parametric statistics as indicated, depending on the normality of the distribution of variables.

Behavioural analysis. Locomotor activity was measured in a circular corridor (Imetronic) ${ }^{13}$. Mice were placed in the corridor $30 \mathrm{~min}$ before injection for the acute experiments. Locomotor sensitization was induced by a single injection of cocaine or morphine ${ }^{13,43}$. Food self-administration experiments were conducted in operant chambers (Imetronic) with two holes, one being the active hole, indicated by a cue light. Nose-poking in the active hole resulted in delivery of a food pellet (Phymep). The light was switched off for $10 \mathrm{~s}$ after the consumption of the food pellet, during which no reward was provided. Mice were fooddeprived $(3.5 \mathrm{~g} / \mathrm{d}$, water ad libitum) starting five days before the experiment, to reach $85 \%$ of initial weight. Self-administration sessions ( $1 \mathrm{~h}$ per day) were conducted five days per week. Mice were trained under a fixed ratio 1 (FR1, 1 pellet per nose poke) schedule. The session was terminated after 100 pellets or 1 h. Acquisition criteria were: stable response with less than $20 \%$ deviation from the mean of the total number of reinforcers earned in three consecutive sessions ( $80 \%$ stability). Criteria were then changed to a fixed ratio 5 (FR5, 1 pellet per 5 nose pokes) schedule. When the $80 \%$ stability criterion had been reached, a reversal procedure was started ( $\mathrm{rFR} 5)$. During reversal the previously active hole became inactive, and vice versa. When the same criteria as above had been met, mice were subjected to a single 2 -h progressive ratio (PR) session in which the requirement to earn a pellet escalated according to the following series: $1,2,3,5$, $12,18,27,40,60,90,135,200,300,450,675,1,000,1,500$. 